\title{
Accurate Estimating Algorithm of Transfer Function for Transformer FRA Diagnosis
}

\section{Document Version}

Accepted author manuscript

Link to publication record in Manchester Research Explorer

\section{Citation for published version (APA):}

Mao, X., Wang, Z., Wang, Z., \& Jarman, P. (2018). Accurate Estimating Algorithm of Transfer Function for Transformer FRA Diagnosis. In 2018 IEEE Power \& Energy Society General Meeting

\section{Published in:}

2018 IEEE Power \& Energy Society General Meeting

\section{Citing this paper}

Please note that where the full-text provided on Manchester Research Explorer is the Author Accepted Manuscript or Proof version this may differ from the final Published version. If citing, it is advised that you check and use the publisher's definitive version.

\section{General rights}

Copyright and moral rights for the publications made accessible in the Research Explorer are retained by the authors and/or other copyright owners and it is a condition of accessing publications that users recognise and abide by the legal requirements associated with these rights.

\section{Takedown policy}

If you believe that this document breaches copyright please refer to the University of Manchester's Takedown Procedures [http://man.ac.uk/04Y6Bo] or contact uml.scholarlycommunications@manchester.ac.uk providing relevant details, so we can investigate your claim.

\section{OPEN ACCESS}




\title{
Accurate Estimating Algorithm of Transfer Function for Transformer FRA Diagnosis
}

\author{
Xiaozhou Mao ${ }^{1}$, Zhongdong Wang ${ }^{1}$, Zanji Wang ${ }^{2}$, Paul Jarman ${ }^{3}$ \\ ${ }^{1}$ School of Electrical and Electronic Engineering, University of Manchester, Manchester, M13 9PL, UK \\ ${ }^{2}$ Department of Electrical Engineering, Tsinghua University, Beijing, 100084, China \\ ${ }^{3}$ The National Grid Company, Warwick, CV34 6DA, UK
}

\begin{abstract}
Frequency response analysis (FRA) is an effective tool to detect winding deformation. If variation occurs to the FRA trace, a fault may be indicated. A new method to estimate the transfer function from FRA measurement results on large bandwidth is proposed. A MATLAB program is developed to auto-process the measured data and to obtain the transfer function expression. Measurement results from large frequency ranges are divided into several ranges, and key information is abstracted from each range to form a new transfer function. Afterwards, correction is still needed to eliminate the difference between estimated transfer function and measured data. This method is verified as valid for estimation of transfer functions of 48 groups of FRA data of a 400/275 kV auto-transformer.
\end{abstract}

Index Terms-Algorithms, FRA, Transfer Function, Power Transformer

\section{INTRODUCTION}

Transformers are very expensive apparatuses of high importance in power systems. Failures of transformers may result in catastrophic consequences and cause huge economic losses. Many in-service transformers have already exceeded their expected usage time [1]. Apart from this aging problem, incidents during transportation or installation and subsequent in-service events may also cause damage to the transformers. Periodic diagnosis or condition-based diagnosis of serving transformer is required to judge heathy condition of the transformers.

Among all categories of transformer failures, winding deformation makes up a considerable percentage [2]. Frequency response analysis (FRA) testing is the technique used to detect the existence of winding deformation. Comparison is supposed to be done between reference FRA data and measured FRA data. Shifting of resonant frequencies is the key feature for FRA to sensitively diagnose the winding deformation. Shifting of higher resonant frequency indicates a smaller change in windings or part of the winding. Shifting of lower resonant frequencies (not affected by core magnetization) indicates a larger change of winding. Analysis methods of FRA data are classified into two types, depending on how the FRA data are dealt with, i.e., the subjective method and the objective method [3]. The subjective method includes visual inspection, difference spectrum and signature spectrum [4]. Compared with subjective methods, objective methods do not rely on expert's experience. Model identification is commonly used in objective methods. Converting FRA data into transfer function expression enables transformer diagnosis and winding model re-construction.

Vector fitting is proposed by Gustavsen and Semlyen, which is widely used for responses function estimation in the frequency domain [5]. It uses partial fraction decomposition of a rational function in the following format:

$$
f(s)=\sum_{n=1}^{N} \frac{c_{n}}{s-a_{n}}+d+s h
$$

where $a_{n}$ are poles which are either real or complex, $c_{n}$ are their corresponding residues, both $d$ and $h$ are real numbers. $a_{n}$ and $c_{n}$ are achieved by renewing the set complex starting poles through least squares approximation. Poles and residues are identified in two different stages. This method is accurate, stable and effective.

The transfer function can be expressed via zeros and poles as follows:

$$
T_{s}=k_{s} \frac{\prod_{k=1}^{N 1}\left(s-z_{r k}\right)}{\prod_{j=1}^{M 1}\left(s-p_{r j}\right)} \times \frac{\prod_{k=1}^{N 2}\left(s-z_{c k}\right)\left(s-z_{c k}^{\prime}\right)}{\prod_{j=1}^{M 2}\left(s-p_{c j}\right)\left(s-p_{c j}^{\prime}\right)}
$$

where $T_{s}$ is the estimated transfer function, $k_{s}$ is the constant coefficient, $z_{r k}$ and $p_{r j}$ are real zero and real pole respectively, and $z_{c k}, z_{c k}^{\prime}$ are conjugate complex zeros, $p_{c j}, p_{c j}^{\prime}$ are conjugate complex poles respectively. The complex zeros and poles are in the form $s=\sigma+j \omega$, where $\omega=2 \pi f$ and $f$ is the frequency location of resonance or anti-resonance, $\sigma$ is frequency dependent damping rate [6]. For real zeros and poles, $\sigma=0$.

A subjective method to estimate transfer function expression of FRA data on wide bandwidth is proposed in this paper by extracting complex zeros and poles from different frequency range, combining them together to form a new transfer function and then correcting the deviation from measured data. This method has a better performance dealing with subtle feature estimation. Also, a MATLAB program is developed to auto-process input data into the parameters of its transfer function expression. 


\section{FRA DATA FROM UK NATIONAL GRID}

FRA data in this paper is provided by UK National Grid, from a 400/275 kV 500MVA auto-transformer, without tap winding, built in 1966 by AEI Wythenshawe. Design has a five limb core and has bolts through the yokes and limbs. Series and common windings are of multiple layer winding type. Fig. 1 is FRA plot tested on B phase High Voltage (HV) winding to Low Voltage (LV) from $0.005 \mathrm{kHz}$ to $200 \mathrm{kHz}$, containing 1120 frequency points. Fluctuations between 0.001 $\mathrm{kHz}$ and $0.1 \mathrm{kHz}$ in phase are caused by noise.

For this transformer, the core dominates up to $2 \mathrm{kHz}$, interaction between windings dominates from $2 \mathrm{kHz}$ to 20 $\mathrm{kHz}$ and winding type dominates from $20 \mathrm{kHz}$ to $200 \mathrm{kHz}$ [3]. A demonstration of how to estimate transfer function will be shown using this FRA plot as an example.
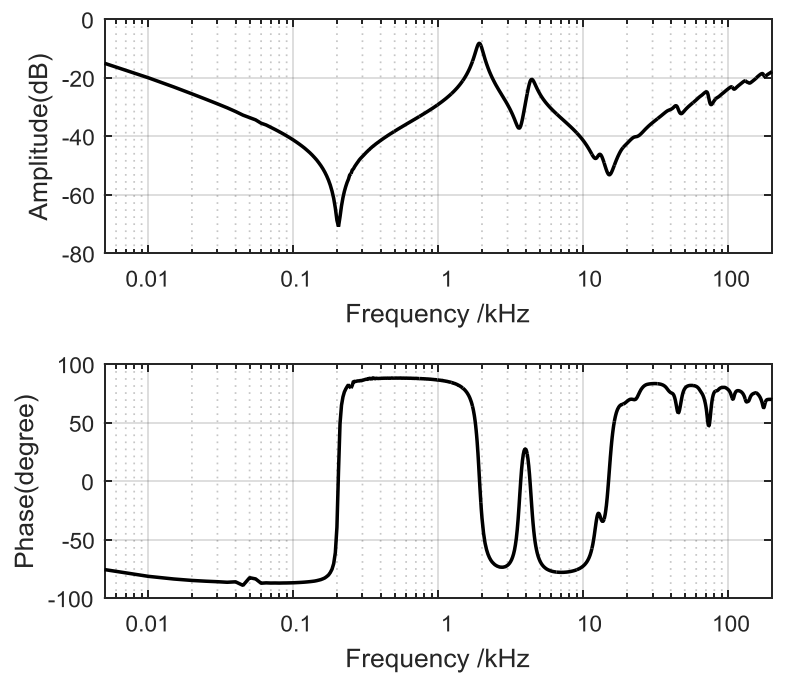

Figure 1. Example of FRA plot tested on B phase HV to LV of a 400/275 kV 500 MVA transformer

\section{Proposed Transfer Function FitTing MEthod}

The proposed method described in this paper can be divided into three steps, and its flow chart is shown in Fig. 2.

MATLAB command 'invfreqs' [7] is used in this method to convert the measured FRA data on small frequency range into a transfer function expression in the continuous frequency domain. The reason why the 'invfreqs' command is not used directly for wide bandwidth is that the quantity of resonances and anti-resonances is large, thus requiring a very high-order transfer function. However, MATLAB can only cope with estimation of relatively low-order transfer function.

\section{A. First Step: Feature Transfer Function}

The first step is to construct a Feature Transfer Function (FTF). There are four stages in this step.

\section{1) Frequency Range Division}

In the first stage, since 'invfreqs' is only capable of extracting a limited amount of zeros and poles, it is necessary to split the measured data into several frequency ranges to ensure that there are not too many data points in each range. Else, some zeros or poles to be estimated may be missing. An example of estimating the whole frequency range transfer function for FRA plot in Fig. 1 will be given. All the 1120 points are re-divided into 7 ranges, i.e., $0.005 \mathrm{kHz}$ to $1 \mathrm{kHz}, 1$ $\mathrm{kHz}$ to $2 \mathrm{kHz}, 2 \mathrm{kHz}$ to $20 \mathrm{kHz}, 20 \mathrm{kHz}$ to $65 \mathrm{kHz}, 65 \mathrm{kHz}$ to $110 \mathrm{kHz}, 110 \mathrm{kHz}$ to $155 \mathrm{kHz}$ and $155 \mathrm{kHz}$ to $200 \mathrm{kHz}$.

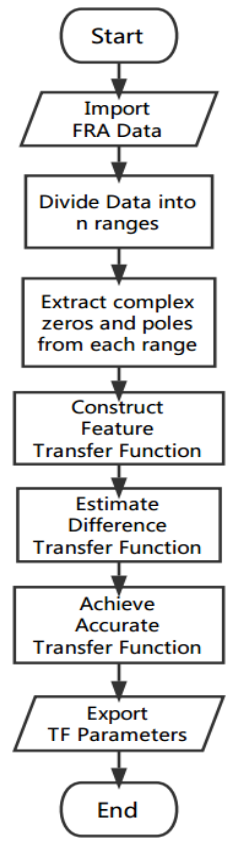

Figure 2. Flow chart of proposed method

\section{2) Selection of Order of Transfer Function}

The order of the estimated transfer function is an input of 'invfreqs', corresponding to the numbers of zeros and poles. When estimating transfer function for each frequency range, the selection of its order will influence the estimation accuracy. If the order of transfer function is less than needed, some plot characteristics may be eliminated. However, there may be redundant zeros and poles when the order is sufficient. The choice of order is not unique. There exists no such relationship that with the increase of the order, accuracy of estimation can be improved. An optimal order should be chosen for each divided frequency range.

The possible range of transfer function order is set according to the approximate number of zeros and poles of each frequency range.For each possible order, the maximum magnitude / phase error is defined as the largest magnitude / phase deviation between measured and estimated data among all the frequency points using 'invfreqs'. The criteria to choose the optimal order from the pre-defined possible order range are as follows:

- The maximum magnitude error of the optimal order should be smaller than 3 times the minimum value of all the maximum magnitude errors from all compared orders. The same rule applies to phase.

- The magnitude correlation coefficient of the optimal order should be larger than 0.9999 .

- The phase correlation coefficient should be as close to 1 as possible. 
An example is given here. The estimated and measured data traces of frequency range $2 \mathrm{kHz}$ to $20 \mathrm{kHz}$ are compared in Fig. 3, after optimal transfer function order 22 is chosen.
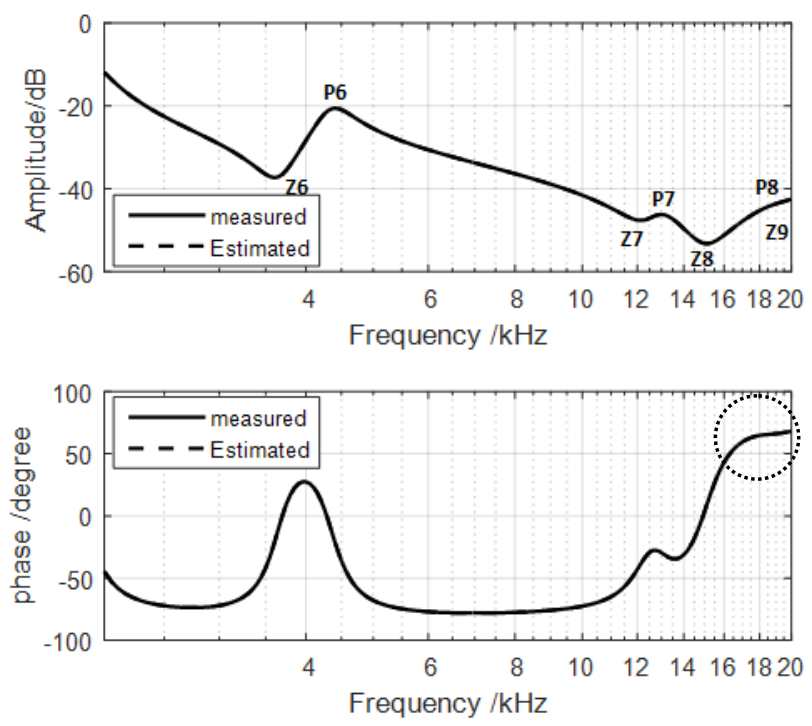

Figure 3. Measured and estimated FRA plot on 2-20kHz

\section{3) Cancellation of Redundant Zeros and Poles}

After an optimal order is selected, redundant zeros and poles should be cancelled. For complex zeros and poles which can be considered redundant, the following criteria apply:

- For the imaginary part, the difference between the frequency location of zeros and poles should be less than $2 / 5$ of the sampling interval;

- For the real part, the absolute difference value should be smaller than 60 or when the absolute difference value is larger than 60 , the percentage of difference should be less than $0.6 \%$.

The first criterion is to make sure that the location of the complex zeros and of the complex poles are close enough to be cancelled, while the second criterion aims to control the damping rate of the zeros and poles to be cancelled. When the frequency is low, the damping rate is small. The situation may occur where the absolute difference of the real part is small, but the percentage difference is large. Thus the second criterion guarantees that the redundant zeros and poles with small real part in low frequency can be cancelled. For real zeros and poles, only the second criterion needs to be met.

Table.1 shows the parameters of transfer function over frequency range $2 \mathrm{kHz}$ to $20 \mathrm{kHz}$, after cancelling redundant zeros and poles. Only the zeros and poles with positive imaginary part are listed in the order of low frequency to high frequency.

\section{4) Extraction of Zeros and Poles}

The last stage of step one is to construct the FTF using the extracted complex conjugate zeros and poles from each range. Each anti-resonance is supposed to correspond to a pair of conjugate complex zeros and each resonance is supposed to correspond to a pair of conjugate complex poles.
TABLE I. ESTIMATED 2-20KHZ TRANSFER FUNCTION PARAMETERS

\begin{tabular}{|c|c|c|c|c|}
\hline & Zero & $\mathbf{f z}(\mathbf{k H z})$ & Pole & $\mathbf{f p}(\mathbf{k H z})$ \\
\hline $\mathbf{1}$ & -244413 & & -108552 & \\
\hline $\mathbf{2}$ & -90929 & & -2314 & \\
\hline $\mathbf{3}$ & 115561 & & 126547 & \\
\hline $\mathbf{4}$ & 188838 & & 152950 & \\
\hline $\mathbf{5}$ & $-1080+413 \mathrm{i}$ & 0.07 & $-717+12025 \mathrm{i}$ & 1.91 \\
\hline $\mathbf{6}$ & $-1381+22869 \mathrm{i}$ & 3.64 & $-1763+27247 \mathrm{i}$ & 4.34 \\
\hline $\mathbf{7}$ & $-5488+77217 \mathrm{i}$ & 12.3 & $-339680+76899 i$ & 12.25 \\
\hline $\mathbf{8}$ & $-6941+94283 \mathrm{i}$ & 15.01 & $-5601+80918 \mathrm{i}$ & 12.89 \\
\hline $\mathbf{9}$ & $-13412+117507 i$ & 18.71 & $-12298+116477 \mathrm{i}$ & 18.55 \\
\hline
\end{tabular}

When extracting key information from each frequency range's transfer function, the two criteria are:

- Only the complex zeros and poles located within the divided small frequency range are selected.

- Complex zeros or poles with ratio of real and imaginary part larger than 2 shall not be selected.

It is found that height caused by complex zeros or poles are decided by the ratio of their real and imaginary parts. When the ratio of real and imaginary parts of a complex zero or pole is larger than 2, the pair of complex zeros or poles can be viewed as a pair of real zeros or poles, the value of which are same as the real part of the complex zeros or poles. Therefore, the second selection criterion is set.

Among all the zeros and poles in Table I, $Z_{6}$ to $Z_{9}$ and $P_{6}$, $P_{8}, P_{9}$ are extracted. The complex pole $P_{7}$ does not correspond to any resonance. It can be seen that $Z_{9}$ and $P_{9}$ are located very closely and the magnitude trace around $18.5 \mathrm{kHz}$ does not show any reflection. However, in Fig. 3 the corresponding range inside the dashed circle on the phase trace shows the minor downward and upward trends, which supports the existence of the complex zeros and poles, and proves that the criteria set is properly.

After applying the criteria above, all selected parameters from seven ranges are listed in Table II. There are 19 complex zeros and 18 complex poles altogether. The constant $k$ is set as 1 here. And the FRA plot, $T_{C}$, formed by those selected complex zeros and poles are shown in Fig. 4.
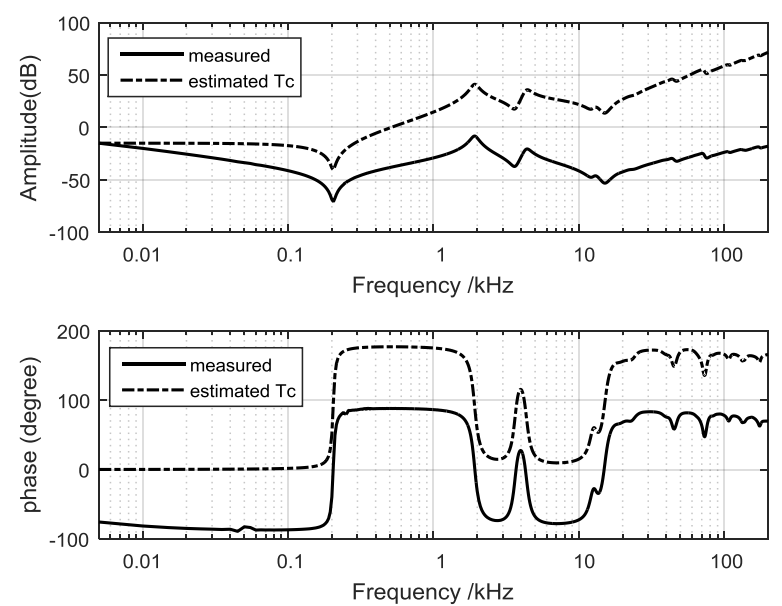

Figure 4. Comparison of FTF Plot and Mesured Data 
TABLE II FTF PARAMETERS

\begin{tabular}{|c|c|c|c|}
\hline \multicolumn{4}{|c|}{$k=1$} \\
\hline Zero & Pole & $\mathrm{fz}(\mathrm{kHz})$ & $\mathrm{fp}(\mathrm{kHz})$ \\
\hline$-33+1282 i$ & $-722+12029 i$ & 0.204 & 1.914 \\
\hline$-1381+22869 i$ & $-1763+27247 i$ & 3.64 & 4.336 \\
\hline$-5488+77217 i$ & $-5601+80918 i$ & 12.289 & 12.878 \\
\hline$-6941+94283 i$ & $-12298+116477 i$ & 15.006 & 18.538 \\
\hline$-13412+117507 i$ & $-13110+146501 i$ & 18.702 & 23.32 \\
\hline$-11946+148301 i$ & $-19154+239420 i$ & 23.6 & 38.1 \\
\hline$-20283+239837 i$ & $-14415+265817 i$ & 38.17 & 42.31 \\
\hline$-13739+264539 i$ & $-17389+280834 i$ & 42.1 & 44.7 \\
\hline$-15783+288870 i$ & $-22272+396277 i$ & 45.98 & 63.07 \\
\hline$-23034+396277 i$ & $-23585+427230 i$ & 63.07 & 68 \\
\hline$-23285+428596 i$ & $-19294+454204 i$ & 68.2 & 72.3 \\
\hline$-19310+466778 i$ & $-22937+519877 i$ & 74.3 & 82.7 \\
\hline$-23621+521010 i$ & $-22932+675461 i$ & 82.9 & 107.5 \\
\hline$-21766+679055 i$ & $-20724+836681 i$ & 108.1 & 133.2 \\
\hline $701958+775922 i$ & $-35857+861613 i$ & 123.5 & 137.1 \\
\hline$-19639+837936 i$ & $899351+885796 i$ & 133.4 & 141 \\
\hline$-34724+866957 i$ & $-30636+1102403 \mathrm{i}$ & 138 & 175.5 \\
\hline$-28682+1106887 i$ & $-83579+1216002 i$ & 176.2 & 193.5 \\
\hline$-80724+1215272 i$ & & 193.4 & \\
\hline
\end{tabular}

It can be seen that there are complex zero and pole located both at $63.07 \mathrm{kHz}$, and their real parts are very similar. However, they cannot be cancelled according to the criteria; otherwise some small difference will be caused both on the magnitude and phase traces.

By this point, the FTF $T_{l}$ has been established as:

$$
T_{1}=\frac{\prod_{k=1}^{n 2}\left(s-z_{c k}\right)\left(s-z_{c k}^{\prime}\right)}{\prod_{j=1}^{m 2}\left(s-p_{c j}\right)\left(s-p_{c j}^{\prime}\right)}
$$

\section{B. Second Step: Difference Transfer Function}

The magnitude and overall plot shape of FTF are not consistent with the measured data as shown in Fig. 4. The second step is to compare FTF with measured data, and the Difference Transfer Function (DTF), representing the deviation, is estimated.

The magnitude data $m_{2}$ and phase data $h_{2}$ of the DTF is calculated as:

$$
\begin{gathered}
m_{2}=\frac{m_{\text {measured }}}{m_{1}} \\
h_{2}=h_{\text {measured }}-h_{1}
\end{gathered}
$$

where $m_{\text {measured }}$ and $h_{\text {measured }}$ are the measured magnitude and phase data, $m_{l}$ and $h_{l}$ are the magnitude and phase of the FTF respectively. The difference data are plotted in solid line in Fig. 4.

When choosing the optimal order of DTF using 'invfreqs', the aforementioned criteria apply, except that the data error for each possible order is ignored in low frequency range because the first few data bits tend to have some large variation while the high frequency range matches very well, especially for phase estimation. This may be caused by the fact that the frequency range is too large. It is within the acceptable range since only one or two data bits are significantly different, which is enlarged in vision due to the logarithmic scale in Fig. 5 .
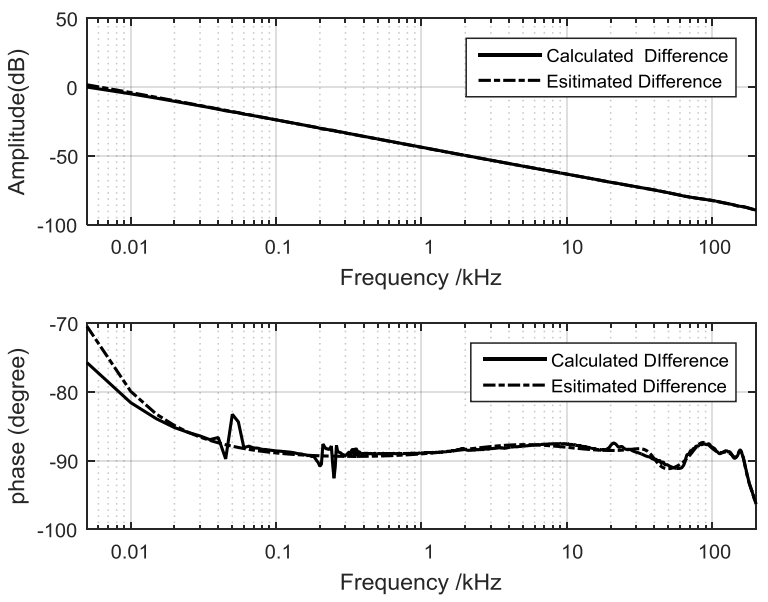

Figure 5. Comparison of DTF Plot and Calculated Data

After the redundant zeros and poles are cancelled from the DTF, Fig. 5 compares the estimated transfer function representing the difference, and Table III lists the parameters of this transfer function in the following format:

$$
T_{2}=k_{s} \frac{\prod_{k=1}^{N 1}\left(s-z_{r k}\right)}{\prod_{j=1}^{M 1}\left(s-p_{r j}\right)} \times \frac{\prod_{k=1}^{n 2^{\prime}}\left(s-z_{c k}\right)\left(s-z_{c k}^{\prime}\right)}{\prod_{j=1}^{m 2^{\prime}}\left(s-p_{c j}\right)\left(s-p_{c j}^{\prime}\right)}
$$

TABLE III. DTF PARAMETERS

\begin{tabular}{|c|c|c|c|}
\hline \multicolumn{4}{|c|}{$k=-3.4369 e-14$} \\
\hline Zero & Pole & $\mathbf{f z}(\mathbf{k H z})$ & $\mathbf{f p}(\mathbf{k H z})$ \\
\hline 3068285 & -11 & & \\
\hline-30400 & -32878 & & \\
\hline$-61165+240663 i$ & $-58293+241886 i$ & 38.3 & 38.5 \\
\hline$-137983+522372 i$ & $-138914+533428 i$ & 83.1 & 84.9 \\
\hline$-69725+872229 i$ & $684191+852497 i$ & 138.8 & 135.7 \\
\hline$-112405+994077 i$ & $-67101+871369 i$ & 158.2 & 138.7 \\
\hline $807949+1028917 i$ & $-109242+1002993 i$ & 163.8 & 159.6 \\
\hline
\end{tabular}

\section{Third Step: Accurate Transfer Function}

The last step is to combine the FTF and the DTF, and the Accurate Transfer Function (ATF) is obtained, which can be viewed as identical to the measured data. The following equation is used to calculate ATF:

$$
T_{s}=T_{1} \times T_{2}
$$

When combining the FTF with the DTF, the constant coefficient of ATF is same as constant coefficient of DTF. All the zeros and poles listed above are contained in the final estimated ATF.

Table IV lists all the parameters used to express the transfer function. For no-source network, zeros and poles of transfer function are expected to appear alternatively. However, for some zeros and poles in Table IV, the sequence is irregular. And such areas can be called fuzzy areas while the remaining areas are called explicit areas. Fig. 6 compares 
the final estimated transfer function traces with measured data, and it can be seen that the result is satisfactory.

TABLE IV. ATF PARAMETERS

\begin{tabular}{|c|c|c|c|c|}
\hline & \multicolumn{4}{|c|}{$k=-3.4369 e-14$} \\
\hline & Zero & Pole & $\mathrm{fz}(\mathrm{kHz})$ & $\mathrm{fp}(\mathrm{kHz})$ \\
\hline 1 & 3068285 & -32878 & 0 & 0 \\
\hline 2 & -30400 & -11 & 0 & 0 \\
\hline 3 & $-33+1282 i$ & $-722+12029 \mathrm{i}$ & 0.204 & 1.914 \\
\hline 4 & $-1381+22869 i$ & $-1763+27247 i$ & 3.64 & 4.336 \\
\hline 5 & $-5488+77217 i$ & $-5601+80918 i$ & 12.289 & 12.88 \\
\hline 6 & $-6941+94283 i$ & $-12298+116477 i$ & 15.01 & 18.54 \\
\hline 7 & $-13412+117507 i$ & $-13110+146501 i$ & 18.7 & 23.32 \\
\hline 8 & $-11946+148301 i$ & $-19154+239420 i$ & 23.6 & 38.1 \\
\hline 9 & $-20283+239837 i$ & $-58293+241886 i$ & 38.17 & 38.5 \\
\hline 10 & $-61165+240663 i$ & $-14415+265817 i$ & 38.3 & 42.3 \\
\hline 11 & $-13739+264539 i$ & $-17389+280834 i$ & 42.1 & 44.7 \\
\hline 12 & $-15783+288870 \mathrm{i}$ & $-22272+396277 i$ & 46 & 63.1 \\
\hline 13 & $-23034+396277 i$ & $-23585+427230 i$ & 63.1 & 68 \\
\hline 14 & $-23285+428596 i$ & $-19294+454204 i$ & 68.2 & 72.3 \\
\hline 15 & $-19310+466778 i$ & $-22937+519877 i$ & 74.3 & 82.7 \\
\hline 16 & $-23621+521010 i$ & $-138914+533428 i$ & 82.9 & 84.9 \\
\hline 17 & $-137983+522372 i$ & $-22932+675461 i$ & 83.1 & 107.5 \\
\hline 18 & $-21766+679055 i$ & $-20724+836681 i$ & 108.1 & 133.2 \\
\hline 19 & $701958+775922 i$ & $684191+852497 i$ & 123.5 & 135.7 \\
\hline 20 & $-19639+837936 i$ & $-35857+861613 i$ & 133.4 & 137.1 \\
\hline 21 & $-34724+866957 i$ & $-67101+871369 i$ & 138 & 138.7 \\
\hline 22 & $-69725+872229 i$ & $899351+885796 i$ & 138.8 & 141 \\
\hline 23 & $-112405+994077 i$ & $-109242+1002993 i$ & 158.2 & 159.6 \\
\hline 24 & $807949+1028917 i$ & $-30636+1102403 i$ & 163.8 & 175.5 \\
\hline 25 & $-28682+1106887 i$ & $-83579+1216002 i$ & 176.2 & 193.5 \\
\hline 26 & $-80724+1215272 i$ & & 193.4 & \\
\hline
\end{tabular}

\section{CONCLUSION}

The method proposed constructs the transfer function by extracting key information from small frequency ranges to construct FTF and correcting it with DTF.

It is accurate and efficient, and the data can be processed automatically by the developed MATLAB program. Compared with other function fitting methods it has a better performance to reflect subtle features.

This method has been tested on eight 400/275 kV autotransformers. For each transformer, six groups of data including three phases, each with two windings, are used - that is 48 groups of data all together. All of the results match well with the measured data, but when tests are done on the tertiary winding, not all of them can find good match. This is because the exact values in set cancellation criteria are related to transformer characteristics, and therefore the values can be changed.

This method can be used for transformer winding diagnosis by comparing the parameters of transfer function representing reference measurement data and the diagnostic measurement data. If the parameters change, a fault may be indicated.
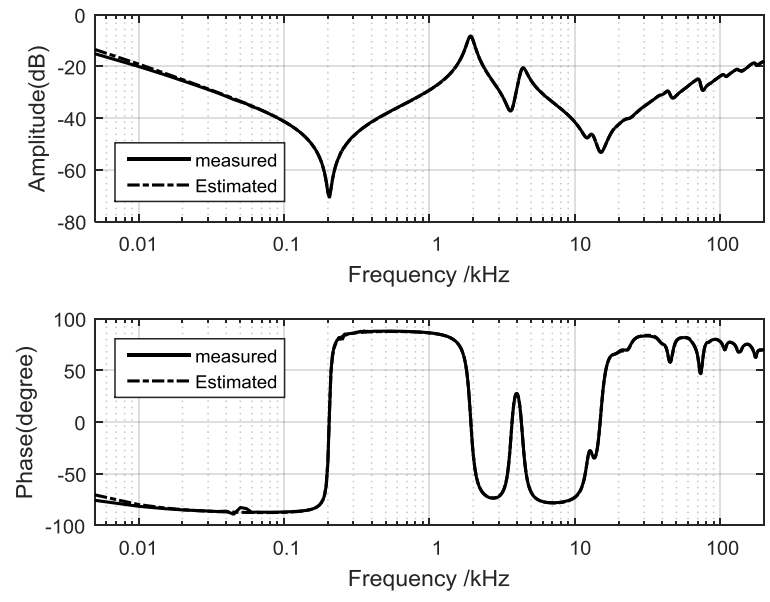

Figure 6. Comparison of ATF Plot and Mesured Data

Besides, the transfer function obtained can be used to design black box model for transformer modeling in power system to investigate the influence of transformers on the system. The model to be designed is capable of simulating the transformers more accurately in wider frequency range.

\section{REFERENCES}

[1] P. Jarman, Z. Wang, Q. Zhong, and T. Ishak, "End-of-life modelling for power transformers in aged power system networks," presented at the CIGRE 6th Southern Africa Regional Conference, Cape Town, South Africa, 2009.

[2] M. Wang, A. J. Vandermaar, and K. D. Srivastava, "Review of condition assessment of power transformers in service," in IEEE Electrical Insulation Magazine, vol. 18, no.6,November/December 2002, pp. 12-25.

[3] Sofian, Dahlina Mohd "Transformer FRA Interpretation for Detection of winding movement", $\mathrm{PhD}$ thesis submitted at the University of Manchester, 2007

[4] S. Birlasekaran and F. Fetherston, "Off/on-line FRA Condition Monitoring Technique for Power Transformer," IEEE Power Engineering Review, pp. 54-56, August 1999.

[5] Gustavsen, B. and Semlyen, A., 1999. Rational approximation of frequency domain responses by vector fitting. IEEE Transactions on power delivery, 14(3), pp.1052-1061.

[6] Yi-ming Zheng, Zan-ji Wang. "Determining the broadband loss characteristics of power transformer based on measured transformer network functions and vector fitting method." IEEE Transactions on Power Delivery 28.4 (2013): 2456-2464

[7] Uk.mathworks.com. (2017). Identify continuous-time filter parameters from frequencyresponse data - MATLAB invfreqs - MathWorks United Kingdom. [online] Available at: https://uk.mathworks.com/help/signal/ref/invfreqs. 